Conference Proceedings Paper

\title{
Evaluation on Topology of Switching Power Amplifier for Active Magnetic Bearings
}

\author{
Yefa Hu, Kezhen Yang, Xinhua Guo, Jian Zhou, Huachun Wu*
}

\begin{abstract}
Switching power amplifier is a key component of the actuator of active magnetic bearing, and its reliability has an important impact on the performance of magnetic bearing system. This paper analyzes the topologies of switching power amplifier of active magnetic bearing. In the case of different coil pair arrangements and bias current distributions, comprehensive evaluation on different topologies of switching power amplifier is introduced. The evaluation has a guiding role in the design of switching power amplifier of active magnetic bearing.
\end{abstract}

Keywords: Active Magnetic Bearing; Switching Power Amplifier; Topology

\section{Introduction}

Active magnetic bearing (AMB) is electromagnetic equipment that can support the high-speed rotating rotor. Compared with traditional bearings, AMB has many unique advantages, such as no contact, no wear, no lubrication, low power consumption and adjustable stiffness and damping [1]. $\mathrm{AMB}$ has a wide application prospect in the fields like uninterruptible power supply (UPS) and satellite control moment gyroscope (CMG). These devices have high requirements for the overall performance and dependability of $\mathrm{AMB}$ system. $\mathrm{AMB}$ is a typical open-loop unstable system, it cannot work normally until feedback control is applied. Its basic principle is shown in Fig.1. The displacement sensor detects the displacement of the rotor off center in two vertical directions and sends it to the controller. The controller calculates and adjusts the reference current signal with an algorithm. The power amplifier and electromagnet are the actuators of AMB. The power amplifier amplifies the reference current signal output by the controller to excite the electromagnet to produce the desired electromagnetic force. Therefore, the performance and reliability of pow er amplifier are of great significance for the stable operation of AMB.

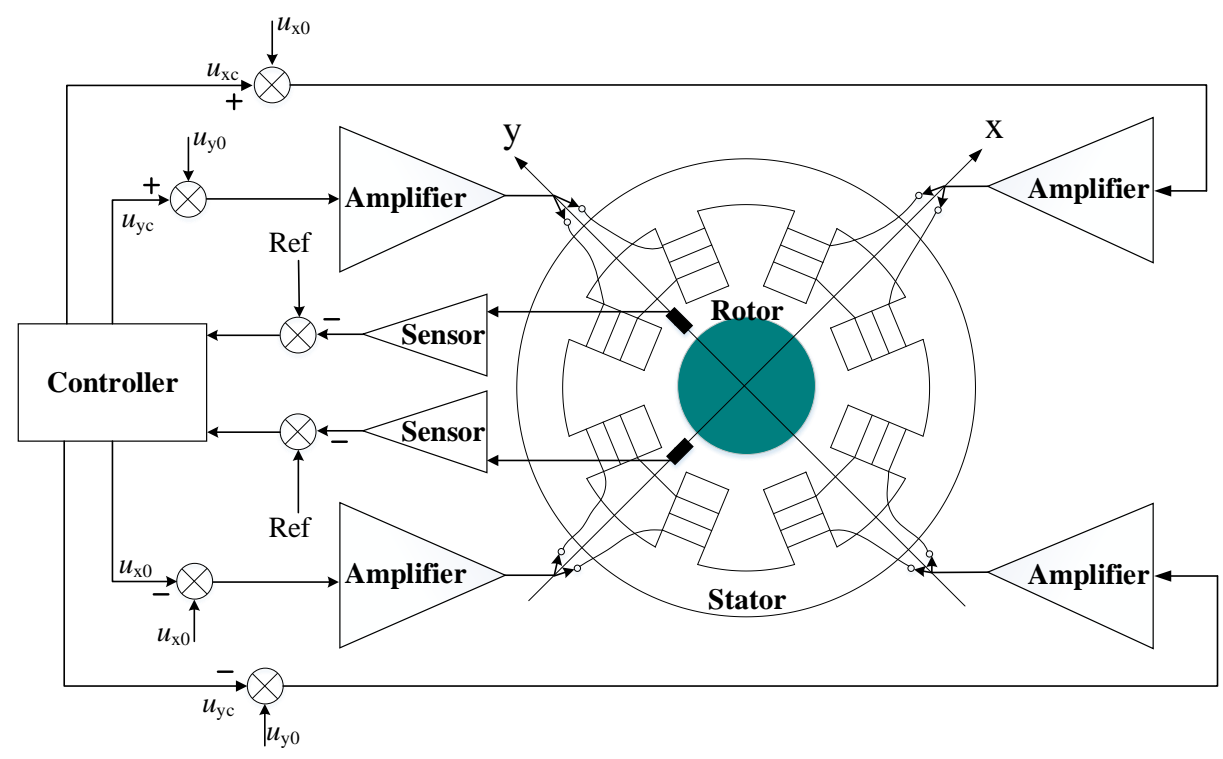

Figure 1. Basic principle of AMB. 
As an important part of magnetic levitation actuator, power amplifier has been concerned and studied by many scholars. The linear power amplifier is the first drive of AMB, but the power consumption of electronic components in the linear region is very large, so the pow er amplifier type has been changed to switching power amplifier (SPA) in the recent 20 years. Xin Cheng et al. studied the accuracy characteristics of a SPA under two-level modulation and analyzed the main factors affecting the inherent current ripple [2]. Yuan Ren et al. proposed a predictive current control method based on the proportional-differential current-sensing resistor netw orks, which solved the problem of digital control delay of the H-bridge power amplifier, reduced the computation load of the algorithm, improved the control accuracy and reduced the current noise [3]. To reduce the influence of phase lag of SPA on system stability and static and dynamic performance of AMB, Jiancheng Fang et al. proposed an adaptive phase-lead compensation strategy based on unsymmetrical current sampling resistance network [4]. Dong Jiang et al. designed a reduced switch converter topology SPA, which reduced the number of switches, gate drivers and PWM signals, and analyzed the modulation methods and the control strategy of the middle bridge arm [5]. Furthermore, Dong Jiang et al. carried out global optimization for the whole system of multi-axis AMBs drive, and proposed a topology of shared-bridge drive with reverse direction, which was then extended to fault-tolerant drive [6]. For the AMBs system with five degrees of freedom (DOF), Jie Zhou et al. designed a SPA with five-phase six-leg topology, and used one-cycle algorithm for control, which effectively reduced the current ripple [7].

However, the research of SPA of AMB at present mainly focuses on the control algorithm to improve the output current accuracy of half-bridge or full-bridge topology power amplifier, and some studies the topology optimization of SPA and puts forw ard the corresponding control method, which significantly reduces the number of components. How ever, the performance evaluation of different topologies is not given, especially in different coil pair arrangements and the distributions of bias current, and needs further study.

Therefore, this paper evaluates different topologies of SPA, discusses SPA performance of different topologies with different coil pair arrangements and bias current distributions, which can provide theoretical guidance and reference for the design of AMB power amplifier, and is of great significance to improve the reliability of pow er amplifier and the wide application of AMB.

\section{Several topologies of SPA}

At present, magnetic bearing SPA generally adopts the bridge topology, and some other topologies are modified based on the full-bridge topology. Fig.2 demonstrates a typical full-bridge topology circuit. The controller controls the opening and closing of four switches through the PWM signal with four isolated drive circuits, and adjusts the current in the coil in real time. Besides, the coil current can be bidirectional. If $S_{1}, S_{2}$, or $S_{3}, S_{4}$ are opened at the same time, the coil current can be increased or decreased under the DC bus voltage $V_{D C}$, and while $S_{1}, S_{3}$, or $S_{2}, S_{4}$ are opened simultaneously the coil current freewheels. Hence the coil current can follow the reference current signal with high performance by controlling the four switches. However, if such a topology is adopted, four power switches and four isolated drive circuits are required for the control of one coil.

In order to improve the support stiffness of $\mathrm{AMB}$, the motion of rotor with a single DOF generally needs two coils with differential control. Therefore, two radial AMBs with two DOF as shown in Fig.1 and one axial AMB with single DOF can form a 5-DOF AMB system to levitate rotor completely. And the rotor can also be supported by only two radial AMBs, and the axial DOF is limited by the coupling connecting the motor to transfer torque, thus a 4-DOF AMB system is built. A 4-DOF AMB system has 8 coils in total and needs 8 full-bridge circuits, as shown in Fig.3, so it needs 32 pow er switches and 32 isolated drive circuits, which greatly increases the failure rate of SPA and reduces the reliability. 


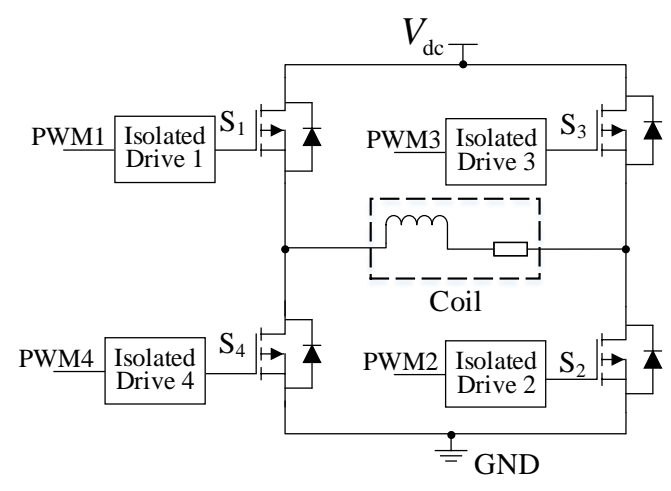

Figure 2. Full-bridge topology circuit of SPA.

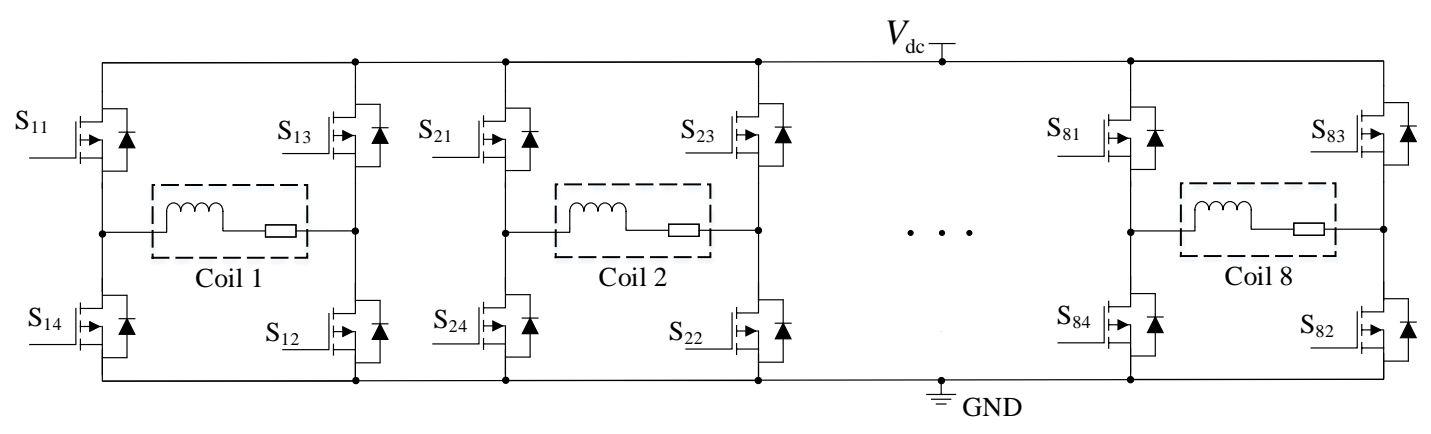

Figure 3. Full-bridge circuit of SPA of 4-DOF AMB.

Because the SPA of AMB employs differential control mode, the winding currents of a pair of coils controlling one axis are equal to the bias current io add and subtract the control current $i_{c}$ respectively, namely changes near the bias current $i_{0}$ as the control current $i_{c}$ changes. Therefore, the winding current only needs to be unidirectional. Saving two switches $S_{3}$ and $S_{4}$ with their driving circuits, the unidirectional current control of one coil is realized by using the half-bridge topology shown in Fig.4. When $S_{1}$ and $S_{2}$ are opened simultaneously, the coil is charged and the current increases; when $S_{1}$ and $S_{2}$ are closed at the same time, the coil discharges through diodes $D_{1}$ and $D_{2}$, and the current decreases; when only one of $S_{1}$ and $S_{2}$ is opened, the coil current will freewheel. This topology can save half of the switches and their driving circuits compared with full-bridgetopology. However, one half-bridge circuit can also control only one coil current, and eight half-bridges are required to achieve complete control for the 4-DOF AMB system, as displayed in Fig.5. Such a large number of components have a high probability of failure, so the dependability of SPA using halfbridge topology is still problematic.

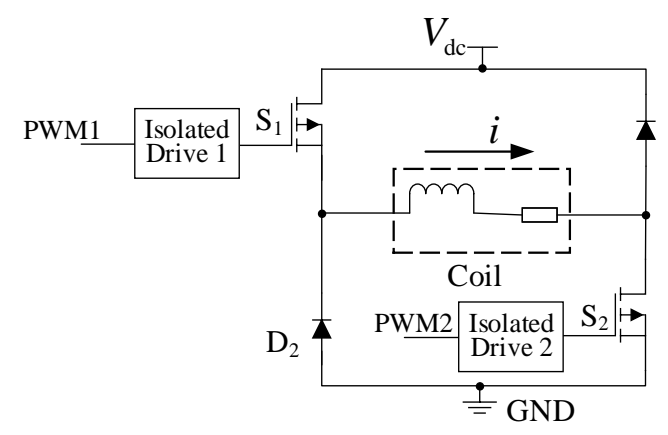

Figure 4. Half-bridge topology of SPA. 


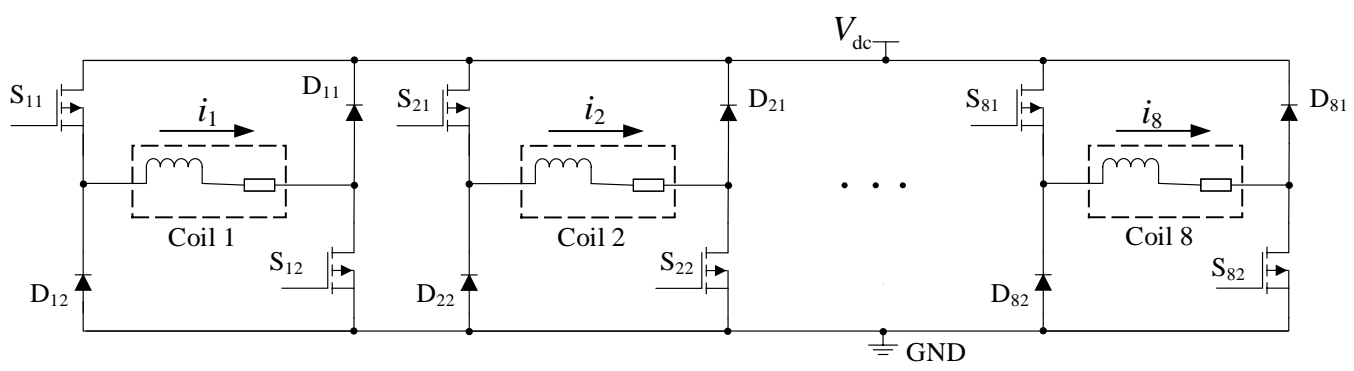

Figure 5. Half-bridge circuit of SPA of 4-DOF AMB.

Considering that a pair of coils on one axis of $\mathrm{AMB}$ adopts differential control and their currents have correlation, two half-bridge circuits controlling two coils can be integrated into one three-phasehalf-bridge circuit, as shown in Fig.6, and Coil 1 and Coil 2 are differential coils. The two coils share a middle phase leg, which reduces the use of switch So' and diode $\mathrm{D}^{\prime}$. Although the position of the switches and freewheeling diodes can be exchanged in principle, the switches $S_{1}$ and $S_{2}$ are generally placed at the low end to simplify the design of pow er circuits of the isolated drive. Furthermore, the middle phase leg switch $S_{0}$ has a fixed duty cycle of $50 \%$ to achieve control simplicity, and the change of coil current is realized by controlling the duty cycle of switches $S_{1}$ and $S_{2}$ on both outer legs [5]. The current passing through the middle phase leg is the sum of the currents of the two coils controlled, namely twice the bias current, which is exactly equal to the extreme current of either outer phase leg. So the components on the middle phase leg do not need special consideration, which is why the two coils controlled by three-phase-half-bridge topology are better to be the two differential coils. How ever, the current flowing through the middle phase leg is constantly twice the bias current, which will bring great challenges to the heat dissipation of the switch $\mathrm{S}_{0}$. Large bias current may cause the switch $S_{0}$ overheated and burn out. Therefore, when selecting the three-phase-half-bridge topology, the bias current, heat dissipation design and switch $S_{0}$ selection should be taken as comprehensive evaluation indexes. When the performance cannot meet the requirements, the ordinary half-bridge topology should be selected.

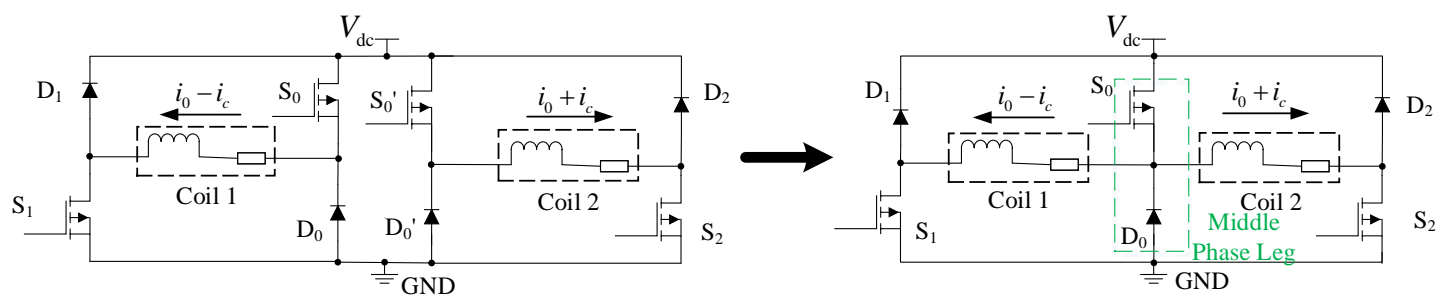

Figure 6. Three-pha se-half-bridge topology of SPA.

When applied to multi-axis AMBs, each middle phase leg of SPA corresponding to each axis plays the same function. By further sharing the middle phase legs and integrating them into a main shared phase leg with a $50 \%$ duty cycle, a more device saving topology is obtained [6]. For a typical 4-DOF AMB, this topology is shown in Fig.7, all coils share the same main middle phase leg. Compared with the traditional half-bridge topology, the number of components required is greatly reduced, which improves dependability of the controller. However, the equipment on the main shared phase leg of this topology must bear a large rated current. The fundamental reason is that all coil currents are in the same direction. According to Kirchhoff's Current Law, the current $I$ on the main shared phase leg is the sum of the currents of the 8 coils:

$$
I=i_{1}+i_{2}+i_{3}+i_{4}+i_{5}+i_{6}+i_{7}+i_{8}
$$

The switch and freewheeling diode which can withstand high current load must be used in the main shared phase leg, and their lives will still be reduced by carryinglarge current. Therefore, the 
reliability of the SPA with shared phase leg topology is much likely to be lower than that with the ordinary half-bridge topology.

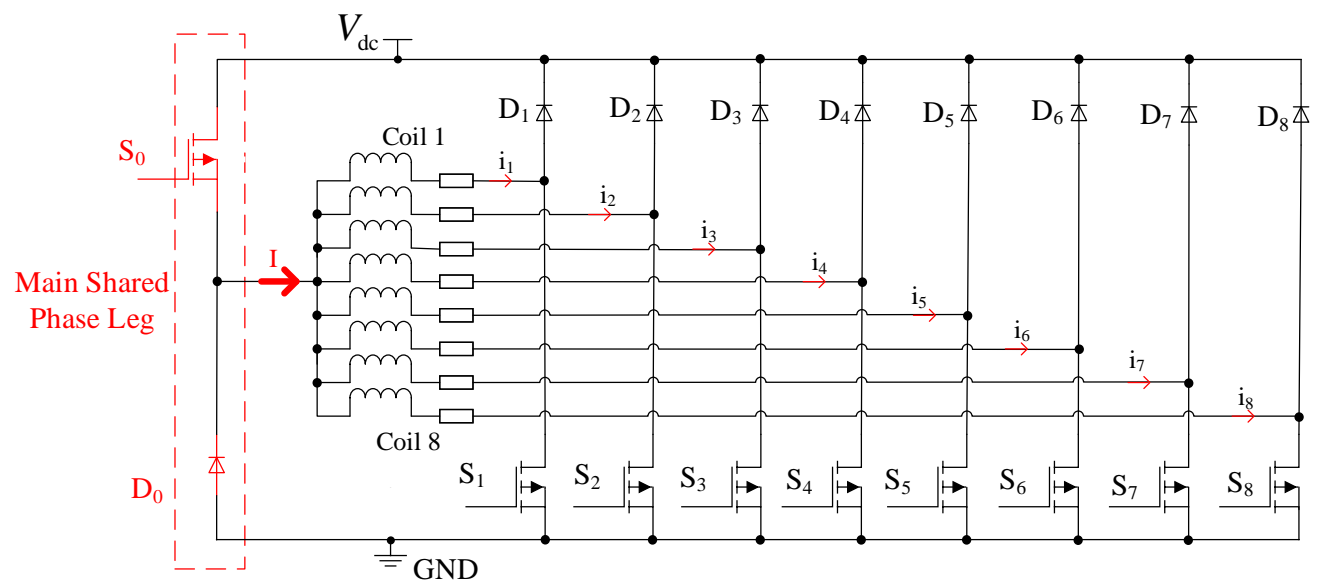

Figure 7. Shared phase leg topology of SPA of 4-DOF AMB.

Since the direction of the coil current does not affect the performance of the AMB, half of the coil currents can be reversed at the cost of adding a switch So' and diode Do', as displayed in Fig.8 [6]. According to the current direction, the coil can be divided into two groups. The current of the first group of coils (Coil 1 to Coil 4) flows from midpoint $\mathrm{O}$, and the current of the second group (Coil 5 to Coil 8) flows to midpoint O. Therefore, according to Kirchhoff's Current Law, the current on the main shared phase leg $I_{\mathrm{N}}$ is the difference betw een the two groups currents instead of the sum:

$$
I_{N}=\left(i_{1}+i_{2}+i_{3}+i_{4}\right)-\left(i_{5}+i_{6}+i_{7}+i_{8}\right)
$$

When the magnetically levitated rotor is supported steadily, the rotor only vibrates in a small range near the balanced position. Therefore, the coil current fluctuates in a small range near the bias current, and the current difference on each coil is very small, the current difference betw een the two groups of coils is also very small. The current of the main shared phase leg is effectively reduced, or neutralized in other words, the current stress and pow er loss decrease efficiently.

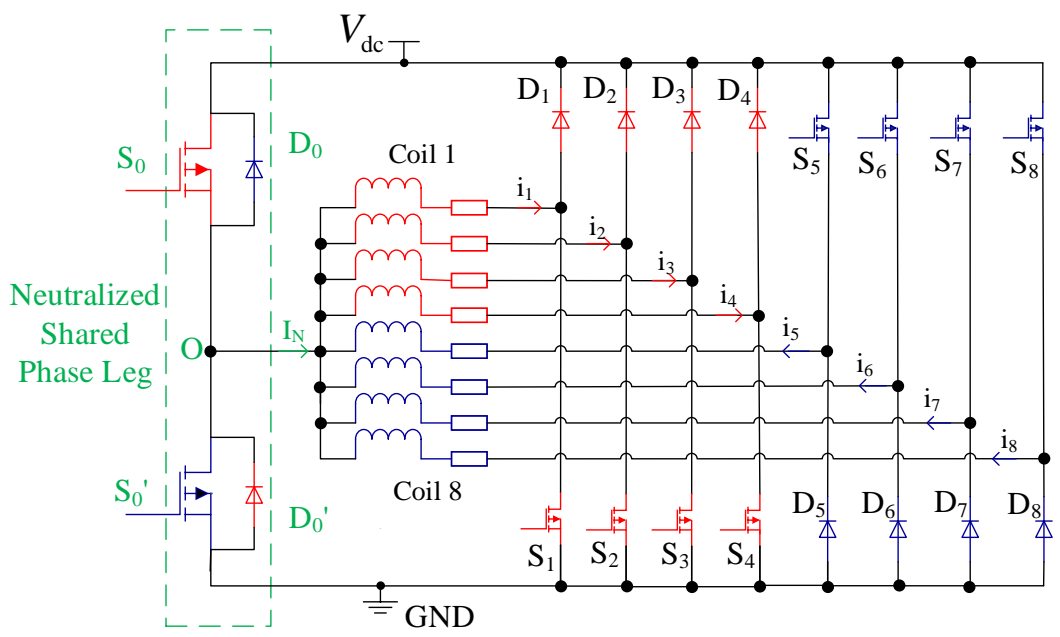

Figure 8. Neutralized shared phase leg topology of SPA of 4-DOF AMB.

The neutralized shared phase leg topology greatly reduces the number of components as well as the current stress of the shared phase leg, which can be considered as a priority in the design of SPA of AMB system. In order to evaluate the SPA topology more comprehensively, this topology is taken as the starting point for more detailed analysis. 


\section{Coil pair arrangement}

When the $\mathrm{AMB}$ is in stable operation, the winding current only follows the control current in a small range near the bias current, so the current difference between the two groups of coils is very small, close to zero. However, when the AMB system is subjected to severe external disturbances such as the base motion, the air gap between the rotor and the stator has a changing trend of high frequency and large amplitude, and SPA needs to adjust the coil current sharply to balance the external disturbance. In this case, for the neutralized shared phase leg topology SPA, the current of each coil has a large change, and it will not change closely near the bias current. Then it is necessary to discuss the coil pair arrangement and evaluate the SPA topology in different forms.

\section{a. Coil pair arrangement of different groups}

For SPA with neutralized shared phase leg topology, when each pair of coils controlling each axis of $\mathrm{AMB}$ are separately arranged in two different groups, and the current in the two coils is apparently the bias current $i_{0}$ add or subtract the control current $i_{c}$, the current stress of the neutralized shared phase leg is analyzed, and the basic parameters of the analyzed SPA are shown in Table 1.

Table 1. Basic parameters of SPA.

\begin{tabular}{|c|c|c|c|c|}
\hline Parameter & $\begin{array}{c}\text { DC Bus } \\
\text { Voltage }\end{array}$ & $\begin{array}{c}\text { Switching } \\
\text { Frequency }\end{array}$ & $\begin{array}{c}\text { Coil } \\
\text { Resistance }\end{array}$ & $\begin{array}{c}\text { Coil } \\
\text { Inductance }\end{array}$ \\
\hline Value & $150 \mathrm{~V}$ & $20 \mathrm{kHz}$ & $2.1 \Omega$ & $5.3 \mathrm{mH}$ \\
\hline
\end{tabular}

Assuming a special case as an example, the bias current $i_{0}=4 \mathrm{~A}$, and the control current of every axis is exactly $i_{c}=2 \mathrm{~A}$ at a certain condition. The coil currents of the first group are all equal to $i_{j}=2 \mathrm{~A}$, $(j=1,2,3,4)$, and the currents of the second group are $i_{j}=6 \mathrm{~A},(j=5,6,7,8)$. Theoretically, the current on the neutralized shared phase leg will reach about $4 \times(6-2) \mathrm{A}=16 \mathrm{~A}$. The numerical simulation results are shown in Fig.9. The simulation results are consistent with the theoretical analysis, which verifies the high current stress of the neutralized shared phase leg. And the current spike is caused by switching noise.

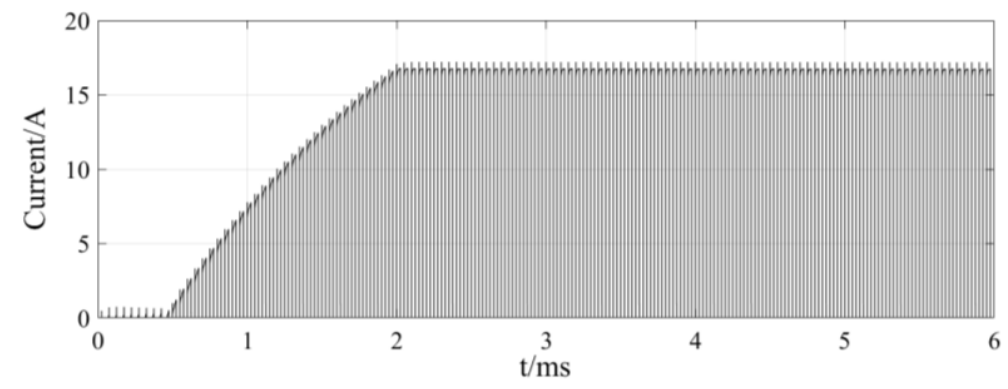

Figure 9. Current stre ss under coil pair arrangement of different groups.

Moreover, this is still not the most severe case. If the external disturbance is particularly large, the current of a pair of coils may reach $0 \mathrm{~A}$ and $8 \mathrm{~A}$ at the bias current of $4 \mathrm{~A}$. Therefore, the current difference between the two groups of coils in the 4-DOF SPA with coil pair arrangement of different groups will be particularly large, and the devices on the neutralized shared phase leg will not only have a short life but also is much likely to fail, which seriously threatening the normal operation of AMB system. To avoid the destructive influence of SPA failure under external disturbance on the stable operation of AMBs, SPA with coil pair arrangement of different groups is not suitable to implement neutralized shared phase leg topology. In order to ensure the reliability of SPA, threephase-half-bridge topology, or even ordinary half-bridge topology is to be considered.

\section{b. Coil pair arrangement of the same group}

When a pair of coils controlling the single axis of AMB are arranged in the same group, a group of coils is composed of two pairs of differential coils. Under the differential control, no matter how 
the air gap betw een the rotor and stator changes, only the control current changes, but the sum of the two coil currents of one axis is equal to twice the bias current. For 4-DOF AMB, four coils of two axes are taken as one group, and four coils of other two axes are the other. Then the current of either group is four times of the bias current io invariably, and the current on the neutralized shared phase leg is pretty small. Under this circumstance, the large current stress of the neutralized shared phase leg caused by severe external disturbance disappears, so the neutralized shared phase leg topology possessing the least components is the best choice of high dependability.

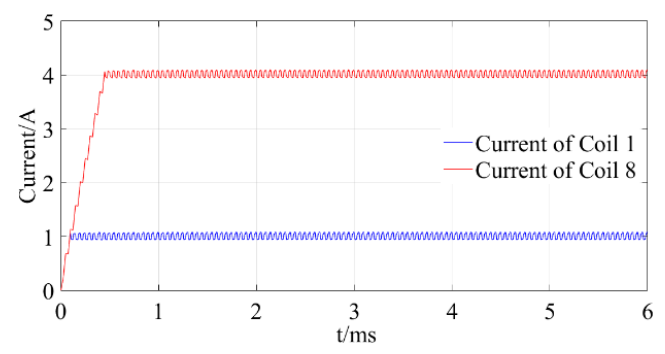

(a)

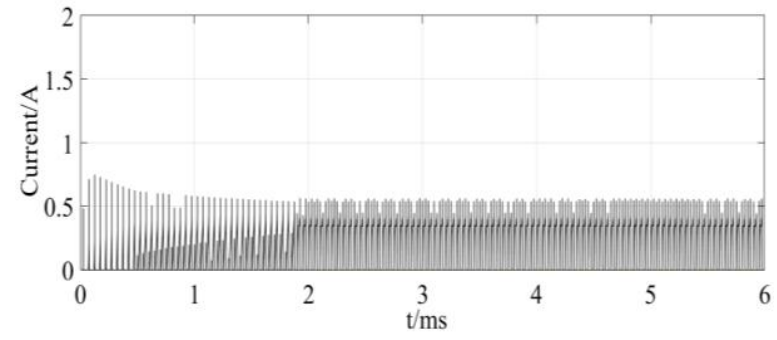

(b)

Figure 10. Performance analysis under coil pair arrangement of the same group.

The performance of the neutralized shared phaseleg topology with coil pair arrangement of the same group is simulated and analyzed. The results are revealed in Fig.10. In order to verify the basic function of SPA with this arrangement of accurately tracking the reference current signal, Coil 1 and Coil 8 are energized with the current of $i_{1}=1 \mathrm{~A}$ and $i_{s}=4 \mathrm{~A}$ respectively, as show $n$ in Fig.10(a). It is seen that SPA can realize the basic function of tracking current. Fig.10(b) show s that when the bias current $i_{0}$ is $4 \mathrm{~A}$, the control current $i_{j}$ of all four pairs of coils is $2 \mathrm{~A},(\mathrm{j}=1,2, \ldots, 8)$, the current on the neutralized shared phase leg is almost equal to zero, and the current stress is greatly relieved, which verifies the analysis. Hence, with the coil pair arrangement of the same group, SPA with neutralized shared phase leg topology has the advantages of stable performance and high reliability, and is the first choice of design.

\section{Bias current distribution}

Although the previous analysis assumes that the bias current of each coil is the same $4 \mathrm{~A}$, in practical application, the bias current of each coil is not necessarily equal to each other. In a 4-DOF $\mathrm{AMB}$ system, the rotation of the magnetically levitated rotor is generally realized by the flexible coupling transferring the torque from the external motor, so the rotor ends can be generally divided into drive-end and non-drive end. The drive-end of the rotor is directly connected to the flexible coupling, which means that the flexible coupling can provide additional stiffness and damping for the drive-end of the rotor. Therefore, under the same working conditions, the electromagnetic force required by the drive-end is less than that of the non-drive end, and the control current of the AMB at the drive-end is less than that at the non-drive end. In the design process of $A M B$, in order to save energy and prolong the life of components, the bias current of $\mathrm{AMB}$ at the drive-end is smaller than that at the non-driveend.

In addition, the bias current of each axis is positively correlated with the anti-interference ability in that direction. The AMB in the direction with large disturbance needs to have larger bias currents to generate greater electromagnetic force to balance the external disturbance. Consequently, in the design process of the AMB system, special attention needs to be paid to the maximum forces required by AMBs in all directions, and then taking it as the design basis of the bias current of each coil in SPA. 


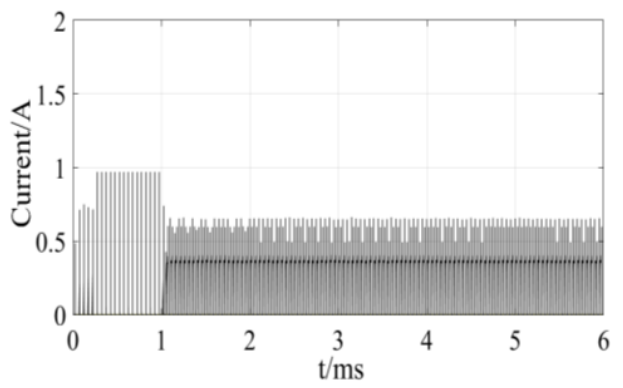

(a)

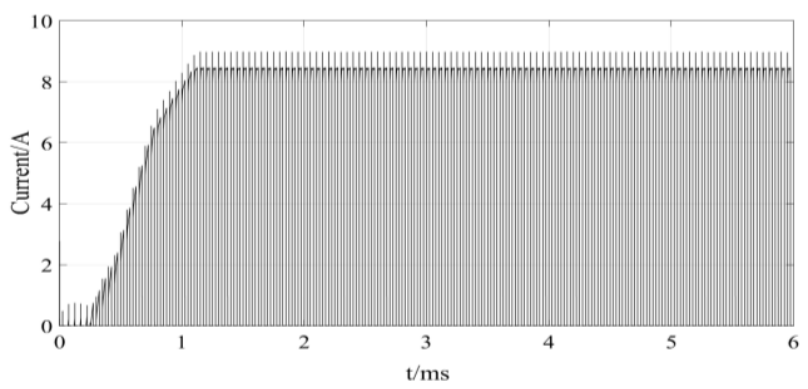

(b)

Figure 11. Current compa rison with diffe rent bias current distributions.

In order to study the influence of different bias current distributions on the performance of neutralized shared phase leg topology SPA, it can be assumed that the bias current of AMB at the drive-end on the $X$ and $Y$-axis coils are set to $1 \mathrm{~A}$ and $2 \mathrm{~A}$ respectively, after considering the influence of coupling and external disturbance, and the bias current of thenon-drive end $\mathrm{AMB}$ on the $\mathrm{X}$ and $\mathrm{Y}$ axis is set to $3 \mathrm{~A}$ and $4 \mathrm{~A}$ respectively. According to the analysis in section 2, the difference of the bias current between the two groups of coils should be as small as possible to mitigate the current stress on the neutralized shared phase leg. Therefore, the coils of $1 \mathrm{~A}$ and $4 \mathrm{~A}$ current are assigned into one group, and the coils of $2 \mathrm{~A}$ and $3 \mathrm{~A}$ current are distributed to the other group. The current on the neutralized shared phase leg is simulated and compared between the two distributions of bias current, as demonstrated in Fig.11.

Fig.11 illustrates that the simulation results are consistent $w$ ith the theoretical analysis. In order to preferentially apply neutralized shared phase leg topology, the coils with unequal bias current should be distributed in the two groups as evenly as possible, and the current stress on the neutralized shared phase leg is the minimum and the SPA has the highest reliability. Furthermore, if the current stress of the neutralized shared phase leg is still too high, the three-phase-half-bridge topology or even the ordinary half-bridge topology should be considered.

\section{Conclusion}

In view of the high-reliability requirement for switching power amplifier of active magnetic bearing, this paper analyzes the different topologies of switching power amplifier. According to the different coil pair arrangements and bias current distributions, the evaluation of different topologies of power amplifier is given combined with the numerical simulation results, which has guiding significance for the selection of power amplifier topology under different conditions, and plays an important rolein promoting the wide application of high performance and high reliability.

Acknowledgments: The project was supported by National Key Research and Development Project of China with grant number 2018YFB2000103.

\section{Reference}

[1] Maslen E H, Schweitzer G, Bleuler H, et al. Magnetic Bearings - Theory, Design, and Application to Rotating Machinery[M]. 2009.

[2] Cheng $X$, Zhang L, Zhou R G, et al.Analysis of output precision characteristics of digital switching power amplifier in the active magnetic bearings system[J].Automatika,2017, 58 (2): 205-215.

[3] Ren Y, Fang J C.Current-Sensing Resistor Design to Include Current Derivative in PWM HBridge Unipolar Switching Power Amplifiers for Magnetic Bearings[J].IEEE Transactions on Industrial Electronics,2012, 59 (12): 4590-4600.

[4] Fang J C, Ren Y.Self-Adaptive Phase-Lead Compensation Based on Unsymmetrical Current Sampling Resistance Network for Magnetic Bearing Switching Power Amplifiers[J].IEEE 
Transactions on Industrial Electronics,2012, 59 (2): 1218-1227.

[5] Jiang D, Kshirsagar P. Analysis and control of a reduced switch converter for active magnetic bearings[C].IEEE Applied Power Electronics Conference \& Exposition,2016:3616-3622.

[6] Jiang D, Li T, Hu Z D, et al.Novel Topologies of Power Electronics Converter as Active Magnetic Bearing Drive[J].IEEE Transactions on Industrial Electronics,2020,67 (2): 950-959.

[7] Zhou J, Deng Z, Liu C, et al. Current ripple analysis of five-phase six-leg switching power amplifiers for magnetic bearing with one-cycle control[C].InternationalConference on Electrical Machines \& Systems,2017.

(C) 2020 by the authors; licensee MDPI, Basel, Switzerland. This article is an open access article distributed under the terms and conditions of the Creative Commons by Attribution (CC-BY) license (http://creativecommons.org/licenses/by/4.0/). 